\title{
Host Galaxy Effects in the Susy Model for Supernovae Ia
}

\author{
L. Clavelli* \\ Dept. of Physics and Astronomy, Tufts University, Medford MA 02155 \\ Dept. of Physics and Astronomy, Univ. of Alabama, Tuscaloosa AL 35487
}

April 22, 2022

\begin{abstract}
For more than forty years virtually all work on the theory of type Ia Supernovae (SN Ia) has assumed that these explosions were due to the transfer of mass to a degenerate star from a partner in a binary system. In these binary models, when the mass of one partner closely approaches the Chandrasekhar maximum for a stable degenerate system, fusion can be initiated and the star explodes. However, a number of long-standing nagging problems and the inability of any specific binary model to fit any significant fraction of SN Ia events suggest that fusion could instead be triggered by a phase transition in a sub-Chandrasekhar white dwarf star. It is possible that remarkable host galaxy effects not considered in previous work on phase transition models could point to a specific source of the supernova trigger. Performing a least $\chi^{2}$ fit to the delay time distribution to fix parameters, we give predictions from the susy phase transition model for the host galaxy effects. In addition we discuss a susy insight into the Phillips relation which is basic to the cosmological importance of the type Ia supernovae.
\end{abstract}

\section{Introduction}

An exhaustive review of the status of binary models for type Ia supernovae has been recently presented in ref. [1]. An essential feature of these models is that accumulation of matter from a partner star leads to an SN Ia explosion near the Chandrasekhar mass, $M_{C}$. A very recent review of all the binary models from the point of view of progenitor identification has also been compiled [2]. A conclusion of this latter review is that no binary model describes a large fraction of the observed SN Ia. In addition, a point made there is that, even if a

*Louis.Clavelli@Tufts.edu, lclavell@ua.edu 
particular progenitor is identified in the future, the question remains as to what happens to the other suggested progenitors as the Chandrasekhar mass is approached. Furthermore, if type Ia supernovae have a variety of initial states, one must explain how a sufficient degree of homogeneity is produced for cosmological applications.

In contradistinction to the binary models we have noted [3] that there are at least six major indications that SN Ia may be due not to mass growth to the Chandrasekhar maximum but, instead, to a phase transition in a possibly isolated white dwarf.

Some years ago, Sim et al. [4] showed that, if a suitable trigger could be found, the detonation of an isolated white dwarf with mass between 0.97 and 1.15 could lead to good agreement with observed SN Ia properties. The model of [5], [6] in which matter at extremely high density undergoes a tunneling phase transition to a background of exact supersymmetry (susy) provides such a trigger. Recently [7] has shown that sub-Chandrasekhar explosions with an undetermined ignition mechanism might also explain the low luminosity supernovae.

The susy model assumes that the exactly supersymmetric universe is the ground state of the multiverse and that the transition rate to this ground state is enhanced at high density. In such a susy background, initial state fermion pairs would convert to boson pairs which, since they are unaffected by the Pauli Principle, would drop to the ground state emitting sufficient energy to trigger fusion in the surrounding matter. Although this model may seem overly radical, if further decades pass without any consensus on a precise binary progenitor, astronomers may want to tolerate some attention to such phase transition models.

There have long been observations relating supernova and host galaxy properties. As indicated in [1] the relations are often of low statistical significance and, in some cases, there are even claims differing in the sense of the correlations. In such a situation it is useful to explore the predictions of the susy model with a view to support or disfavor the model once definitive data become available. In the previous studies of the susy model cited above, there was no consideration of the supernova-host-galaxy correlations which are taken up in the present article. In the binary models, the host-galaxy effects could be related to effects on the rate of mass growth although chemical composition effects in binary models have also been proposed [8]. Some of the predictions of this latter study are, however, not in full agreement with data. For instance, the binary model predicts a rapidly decreasing nickel mass as a function of metallicity while the data shows constant or increasing mass in $\mathrm{Ni}^{56}$. If, on the other hand, an isolated dwarf can explode as in the phase transition models, the host-galaxy effects must be due to the composition of the dwarf and its relation to galactic age and metallicity.

In the susy model, as laid out in [5], 6], and [3], every white dwarf has a natural massdependent lifetime. In a restricted range of mass, the lifetime ranges from a small fraction of a gigayear to gigayear scales. Below a certain mass, the lifetime of the white dwarf is longer than the current age of the universe so it is effectively stable. In this model, the delay time distribution (DTD) from birth of the white dwarf when fusion ceases to its supernova explosion and the ejected mass distribution (EJMD) are foldings of the white dwarf mass 
distribution with a phase transition probability. Each of these could be metallicity dependent as discussed below but only the latter is an intrinsic property of the susy model.

In the binary models, the DTD and EJMD are multi-parameter functions of the binary mass distribution and orbital parameters. At present there are few observational constraints on these parameters so predictions are dependent on model dependent simulations. A prime parameter is the fraction of white dwarfs in binary systems. In the double degenerate (DD) scenario this fraction must, most likely [1], be above 50\% although no high mass binary white dwarf systems have as yet been observed. If this test is passed, if the other counterindications [3] to binary models are overcome, and if the fits to the DTD and EJMD are equal or better than those of the phase transition model, one might prefer the binary model as possibly being simpler.

In the following section we discuss the composition of the white dwarfs and their mass distribution.

\section{The White Dwarf Mass Distribution}

In the phase transition model the rates are proportional to the single white dwarf production rate for which good data are available. White dwarf binaries, in principle, are counted twice in this distribution.

The Salpeter initial mass function with a linear relation between the main sequence mass and the resulting white dwarf mass gives an excellent fit to the observed hot white dwarf mass distribution between the peak at $0.6 M_{\odot}$ and $0.8 M_{\odot}$ as shown, for example, in [6]. The hot white dwarf mass distribution is taken to approximate the birth mass distribution. However, as can be seen in fig.1, the Salpeter initial mass function overpredicts the white dwarf mass distribution for masses above 0.8. The white dwarf mass is a monotonically increasing function of the mass of the parent main-sequence star [9]. We assume, as seems reasonable, that the higher mass parent stars are of higher temperature and hence burn to elements of higher atomic number. These higher $Z$ elements are then passed on to the white dwarf as well as to the ambient interstellar medium. Higher mass white dwarfs are, therefore, associated with higher metallicity environments and are produced less efficiently in low metallicity environments leading to the deficit relative to the solid curve seen in fig.1. Main sequence stars in the range from 8 to 11 solar masses produce white dwarfs [10], [11] that are primarily oxygen-neon-magnesium mixtures as reviewed, for example, in [12]. Main sequence stars below 8 solar masses produce mainly carbon-oxygen dwarfs with masses below $0.9 M_{\odot}$ which form the great majority of observed white dwarfs. White dwarfs near the Chandrasekhar mass may also have an iron core [13], [14], 15].

The "metallicity" in a stellar neighborhood is a variously defined measure of the amount of higher atomic number elements in the system. A commonly used single parameter measure, 


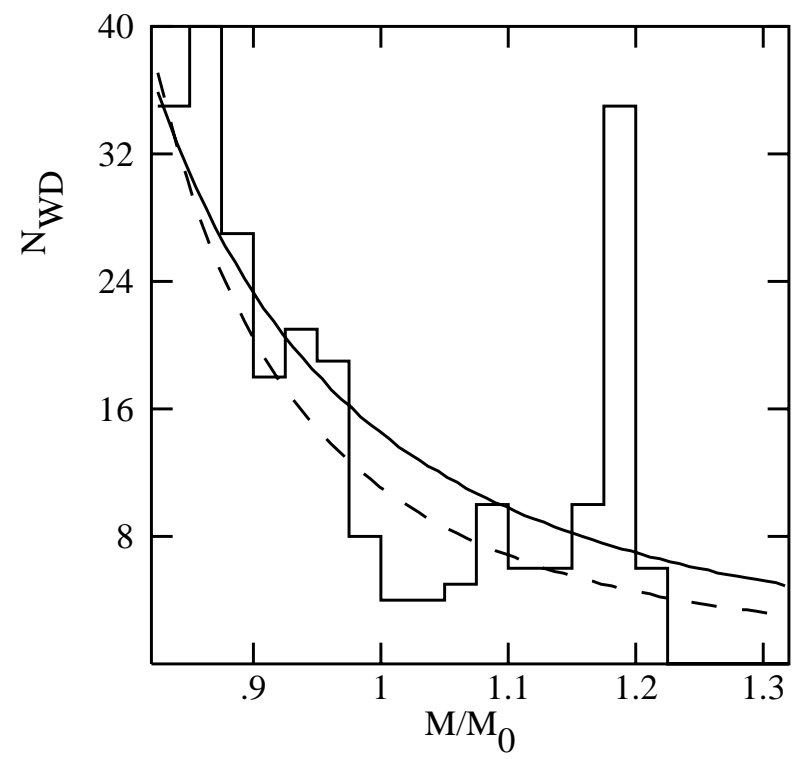

Figure 1: White Dwarf production rate for average to high metallicity environments (solid curve) and low metallicity environments (dashed curve) compared to white dwarf masses as observed in the low metallicity parts of the Milky Way. The solid curve is an expanded view of the high mass tail of the full Salpeter type fit to the white dwarf mass distribution shown in ref. [6] .

normalized to zero for the sun, is

$$
[F e / H]=\log _{10}\left(f_{26} / f_{1}\right)-\log _{10}\left(f_{26} / f_{1}\right)_{\odot}
$$

Here, $f_{Z}$ is the fraction of atoms having atomic number $Z$. The solar abundances are such that the second term has the value 0.02. $[\mathrm{Fe} / \mathrm{H}]$ is a convenient proxy for a more physically relevant expression which must depend on other heavy elements in addition to iron. Since iron is inert to fusion, the expression eq.2.1 nevertheless highlights the expectation that, if fusion is the sole energy source, the nickel production and supernova energy release decrease with heavy element abundance contrary to empirical indications (See, for example, figure 2 of [8]).

In the thin disk of the Milky Way, which includes the sun, $-0.5<[\mathrm{Fe} / \mathrm{H}]<0.3$ while in the thick disk $[\mathrm{Fe} / \mathrm{H}]$ is typically lower [16]. In the central bulge of the Milky Way and in galaxy clusters higher metallicities are plentiful due, presumably, to a rich history 
of supernovae which efficiently convert low mass elements to high mass elements and seed the environment with elements of high atomic number. Mathematically, $[\mathrm{Fe} / \mathrm{H}]$ ranges over the entire real axis as the fraction of iron or hydrogen goes to zero while the atomic number averages are positive and limited.

High concentrations of iron are typically produced together with high concentrations of other high $\mathrm{Z}$ elements. $[\mathrm{Fe} / \mathrm{H}]$ thus monotonically increases with the logarithm of the average atomic number of elements in a galactic neighborhood.

$$
\bar{Z}=\sum Z_{i} f_{i}
$$

In a toy galaxy containing only hydrogen and a small amount of iron, $[\mathrm{Fe} / \mathrm{H}]$ is related to the average atomic number $\bar{Z}$ in the environment by

$$
[F e / H]=\log \frac{f_{26}}{1-f_{26}}+0.02 \approx \log \frac{\bar{Z}-1}{25}+0.02
$$

The average heavy element atomic number of a main sequence star has, initially, a term equal to that number in the environment. The heavier elements should condense at the center and be preferentially passed on to the daughter white dwarf. Thus, while the heavy element fraction in a stellar neighborhood would be expected to vary linearly as a function of the galactic age at stellar birth, the mean atomic numbers in the white dwarf should vary more than linearly due to the central location of the dwarf at birth. These processes could lead to the correlations noted above [10], [11, [13], [14].

In summary, white dwarfs with masses above 1.0 will have an average constituent atomic number above that of oxygen and are produced preferentially in high metallicity environments whereas most of the observed white dwarfs have a $\mathrm{C}$-O composition and come from low metallicity environments.

We normalize the white dwarf production rate assuming a constant star production rate for 12.8 Gyr. There is, of course, some variation in this number and the rate would be greater in star-burst galaxies. The Salpeter initial mass function for a main sequence star falls off as the power 2.35. If there is a linear dependence of the white dwarf mass, $M(0)$, as a function of the parent main sequence stellar mass. we would expect the white dwarf production rate to vary as

$$
F(M(0))=a_{0}\left(\left(M(0)-b_{1}\right) / c_{1}\right)^{-2.35} \quad M(0)>0.62 M_{\odot}
$$

with

$$
a_{0}=5.48 \frac{12.8 G y r}{t_{g}} \mathrm{WD} / \mathrm{yr} / 10^{10} \mathrm{WD}
$$

For $0.6<M(0)<1.35$, the fit shown in ref. [6], as appropriate to the birth mass distribution in some average metallicity environment and, correspondingly, in some average 
galactic age $t_{g}$ took

$$
b_{1}=0.478 \quad c_{1}=0.0903
$$

In the present fit we take a nominal age of $t_{g}=12.8 \mathrm{Gyr}$ to fit the data in the delay time distribution (DTD). Good fits can also be found with ages ten to twenty percent lower. With future high statistics data it should be possible to study the DTD as a function of the host galaxy age and metallicity.

In lower metallicity environments the rough fit of fig.1 1 shown in the dashed curve uses

$$
b_{1}=0.59 \quad c_{1}=0.064
$$

which comes from the crude fit shown in the dashed curve of fig. 10 to the tail of the observed distribution of local white dwarfs . The spike at $M(0)=1.2$ is known to be an artifact of the data treatment at high masses and should be ignored [17] or spread among higher masses. Other authors discussing the variation of the initial mass function are refs. [18, [19, and [20]. The white dwarf sample in the histogram of fig. 1] comes from the local low metallicity environment whereas the Salpeter fit shown in the solid line derives primarily from high mass main sequence stars and their supernovae which are clustered at about redshift 0.5 and which preferentially produce the high mass dwarfs. Since we are primarily interested in the sense of the metallicity dependence and only secondarily in its magnitude, we do not attempt to define an explicit $\mathrm{Z}$ dependence for $b_{1}$ and $c_{1}$. Roughly speaking, in advance of more precisely discriminating data, we consider negative $[\mathrm{Fe} / \mathrm{H}]$ as low multiplicity and $[\mathrm{Fe} / \mathrm{H}]>0.5$ as high metallicity.

\section{Phase Transition Probability in the Susy Model}

If the ground state of the universe is exactly supersymmetric and the current state is one of broken supersymmetry, the probability per unit space time volume of vacuum decay to the ground state in interstellar space is given by the Coleman-DeLuccia [21] formula:

$$
\frac{d^{2} P}{d t d^{3} x}=A e^{-B}
$$

where the action takes the form

$$
B=\frac{27 \pi^{2} S^{4}}{2 \hbar c \bar{\epsilon}^{3}} .
$$

$S$ is the surface tension of a critical bubble of true vacuum nucleated in the ambient false vacuum universe and $\bar{\epsilon}$ is the average energy density difference between the two vacua. Thus, given a knowledge of $\bar{\epsilon}$, the transition probability depends on the two parameters $S$ with 
units of energy per unit area and $A$ with units of an inverse space time volume. In the phase transition theory bubbles of all radii are constantly being nucleated in the false vacuum but most of them will be immediatey quenched in a competition between the volume energy, $4 \pi r^{3} \bar{\epsilon} / 3$, tending to expand the bubble and the surface energy, $4 \pi r^{2} S$, tending to collapse it. In a homogeneous vacuum, where the energy difference is equal to its average, bubbles with initial radius greater than a critical value

$$
R_{c}=\frac{3 S}{\bar{\epsilon}}
$$

will grow at the speed of light to complete the vacuum transition. Presumably, the bubble growth speed and the speed of light in dense matter are also equal.

\section{Density Enhancement of the Susy Phase Transition}

Although it is proven only in lower dimensions [22] [23], it is reasonable to assume that, in a dense medium, the phase transition is accelerated. This can be naturally implemented by assuming that, in the above formulae, the vacuum energy difference is replaced by the total energy difference. Thus, in dense media, the lifetime $\tau(M)$ for a white dwarf of mass $M$ is given by

$$
\frac{d P}{d t}=A V(M) \equiv \frac{1}{\tau(M)}
$$

where

$$
V(M)=\int d^{3} x e^{-B}
$$

and the action now takes the form

$$
B=\frac{13.5 \pi^{2} S^{4}}{\hbar c(\bar{\epsilon}+\overline{\Delta \rho(r)})^{3}}
$$

The critical radius or minimum radius of a successful bubble nucleation is

$$
R_{c}=\frac{3 S}{\bar{\epsilon}+\overline{\Delta \rho}}
$$

Without loss of generality we can factor out of $A$ the inverse of the maximum of $V(M)$ over all white dwarf masses leaving a free parameter with dimensions of inverse time.

$$
\frac{d P}{d t}=\frac{1}{\tau_{0}} \frac{V(M)}{V_{\max }} \equiv \frac{1}{\tau(M)}
$$


The free parameter $\tau_{0}$ becomes then the minimum lifetime of the dwarfs in the sample. If $\Delta \rho$ is simply and universally proportional to the density as assumed in earlier work we can define a critical density $\rho_{c}$ such that, in dense matter where $\bar{\epsilon}$ is negligible,

$$
B=\left(\frac{\rho_{c}}{\rho}\right)^{3}
$$

In white dwarf physics the natural scale of energy is the solar mass, $M_{\odot}$, and the natural scale of distance is the Earth radius, $R_{E}$. In ref. [6], a good fit to the delay time distribution was found with $\rho_{c}=7.42 \cdot 10^{7} \mathrm{~g} / \mathrm{cm}^{3}=9.69 M_{\odot} / R_{E}{ }^{3}$. With this parameter value, the surface tension is such that the critical radius in empty space is of the order of galactic size making our universe safe for billions of additional years. In an inhomogeneous medium, the growth of a critically sized bubble will be halted if the critical radius of eq.4.14 becomes greater than the current bubble radius. In an uncompactified superstring theory or one compactified on tori the ground state vacuum energy density vanishes so that the energy density difference is equal to the vacuum energy density in the broken susy phase which is currently measured to be

$$
\epsilon=3.66 \mathrm{GeV} / \mathrm{m}^{3}
$$

\section{Metallicity Dependence of the Susy Phase Transition}

In the susy phase transition model the energy density difference in dense matter is the energy trapped in high energy levels due to the Pauli Principle. This energy is released in a transition to exact susy since Fermion pairs can convert to pairs of degenerate Bosons. The conversion takes place in nuclei on strong interaction time scales $\left(\approx 10^{-24} s\right)$ via gluino exchange between quarks. On the electromagnetic time scale $\left(\approx 10^{-6} \mathrm{~s}\right)$ electron pairs can convert into selectron pairs via photino exchange. The former conversion has a greater energy release but does not immediately affect the electron degeneracy pressure which supports the star.

The energy release per unit mass from electron to selectron conversion is equal to the electron internal energy that would be released as the star explodes. The electron to selectron conversion in a susy core, therefore, provides no additional energy release relative to the standard model once the explosion is triggered. Thus, the energy provided in the susy phase transition model can be considered as coming entirely from the collapse of the Pauli towers in nuclei. The first estimates based on the Fermi gas model indicated $\Delta \rho \approx 0.02 \rho$ some twenty times greater than the fusion energy released in carbon. In the Fermi gas model, the Pauli energy is primarily a function only of the atomic number to atomic weight ratio, $Z / A$. Consequently the energy release would be the same for the all the low-lying stable nuclei carbon, oxygen, neon, and magnesium. This leads to a universal form for the action, $B$. However, the Fermi gas model fails to predict the magic numbers in nuclei which are naturally accommodated in the nuclear shell model. As we will discuss in this article, the 
nuclear shell model in combination with the phase transition model does predict atomic number dependence and allows for the correct sense of the SN Ia-host-galaxy correlation.

The primary host galaxy properties of interest in supernova physics are:

1. metallicity

2. galaxy age

3. galaxy mass

4. star formation rate

The itemized galactic properties are strongly interrelated. It can be argued that high mass galaxies have a greater number of high mass stars which will produce a greater number of high mass white dwarfs. Since elements of high atomic number are produced in supernovae, older galaxies with a longer history of supernovae are expected to have higher metallicity. These higher metallicities are then preferentially passed on to new stars in those galaxies. Old stars, necessarily produced in old galaxies, have low metallicity. The average atomic number in a white dwarf star should be an increasing function of the metallicity of its environment at the time of birth.

In the susy phase transition model each white dwarf has a mass dependent lifetime as given in eq.4.11, In a star-burst galaxy, the supernova rate will preferentially come from stars with masses near the minimum lifetime. Only when the star formation rate slows will stars with other masses contribute proportionately to the supernova rate.

The above mentioned galactic properties have well established correlations with the SN Ia rate and the $\mathrm{SN}$ Ia peak brightness or $\mathrm{Ni}^{56}$ production.

\begin{tabular}{|c|c|c|c|c|}
\hline $\mathrm{Z}$ & & $\mathrm{Ni}^{56}$ & $\mathrm{Si}^{28}$ & mean \\
\hline 6 & carbon fusion & 0.00104 & 0.00081 & 0.00099 \\
\hline 8 & oxygen fusion & 0.00072 & 0.00051 & 0.00068 \\
\hline 10 & neon fusion & 0.00064 & 0.00042 & 0.00059 \\
\hline 12 & magnesium fusion & 0.00041 & 0.00020 & 0.00037 \\
\hline
\end{tabular}

Table 1: fraction of rest energy released in fusion reactions to $\mathrm{Ni}^{56}$ and intermediate mass elements (IME) represented by $\mathrm{Si}^{28}$. The last column gives the mean energy release per unit mass assuming equal production of $\mathrm{Ni}^{56}$ and IME.

Each nickel nucleus decays to iron with a characteristic energy release which we neglect so the remaining energy release corresponds to that after standardization.

The best linear fit to the energy released in fusion to masses $M\left(N i^{56}\right)$ and $M\left(S i^{28}\right)$ is

$$
E_{f u s}=(15.86-0.98 Z) M\left(N i^{56}\right)+(13.43-0.95 Z) M\left(S i^{28}\right)
$$

The observational results of ref. [24], noted also in [8], are that a mass of $N i^{56}$ of about $0.55 M_{\odot}$ is produced in normal SN Ia roughly independent of metallicity though with large 
errors. This estimate is also consistent with the observations of [25] which, however, show some positive correlation of the nickel mass with the ejected mass. Ref. [26] supports a $\mathrm{Ni}^{56}$ production of about half of the ejected mass, $M$. For definiteness, we adopt, following [26],

$$
M\left(N i^{56}\right)=0.5 M
$$

We also assume slightly less than this in intermediate mass elements, represented by $S i^{28}$

$$
M\left(S i^{28}\right)=0.45 M
$$

This leaves an unburned mass

$$
M_{\text {unburned }} \approx 0.05 M
$$

The theoretical result of ref. [6] is that $M$ is bounded below by about $0.95 M_{\odot}$.

The fact that the fusion energy available is maximized in carbon has suggested that, in the binary models, white dwarfs with a higher percentage of carbon, as would be obtained in low metallicity environments, would have an increased production of nickel and, therefore, a greater luminosity [27] but there are counter-arguments to this [28].

It is commonly thought that the progenitors of normal type Ia supernovae are carbonoxygen mixtures not because of any direct evidence [29] but because such mixtures lead to more successful standard model simulations once fusion is somehow triggered. As seen for example in [3], a roughly fifty-fifty mixture of carbon/oxygen fusing to a roughly fifty-fifty mixture of iron group elements and intermediate mass elements will produce enough fusion energy to match experiment. In this case the maximum amount of energy input from a source beyond the standard model is sharply limited although some such energy input is needed to trigger fusion. Typical white dwarfs will have maximum temperatures and pressures several orders of magnitude below that at which nuclei will touch and ignite fusion. Although, in [6], no assumption was made as to the atomic composition of the white dwarfs undergoing supernova, the progenitor (and ejected) mass was found to be in the range of O-Ne-Mg white dwarfs.

In fact, an energy deficit in the standard model begins to open up if there are unburned remnants or if the initial carbon composition falls below about 0.5. Such low carbon content of at least some white dwarf stars is suggested by recent asteroseimological analyses [30]. Since, contrary to expectations from simulations, a large central oxygen content was found even for a light $\left(0.5 M_{\odot}\right)$ white dwarf, it would not be surprising if, in heavy white dwarfs, a large $\mathrm{O}-\mathrm{Ne}-\mathrm{Mg}$ concentration becomes also observationally established. Furthermore, unburned oxygen is observed in SN Ia but, normally, no unburned carbon. This might be the first reason to doubt the progenitor identification as a $\mathrm{C}-\mathrm{O}$ mixture. The lighter elements in a mixture, preferentially found in the low density outer regions of the star, are the most likely unburned ejecta. Any significant amount of unburned elements, of course, reduces the fusion energy output and causes additional strain on the standard model. Further supporting evidence comes from ref. [31] which finds that among carbon-oxygen white dwarfs the 
oxygen content near the center is nearly $80 \%$ with the carbon content increasing toward the outer strata and these white dwarfs come from main sequence stars in the 5 to 7 solar mass range.

In the phase transition model [6] the progenitor mass ranges roughly from roughly $M_{\odot}$ to $1.4 M_{\odot}$ which, neglecting a small $(\approx 0.1 \%)$ surviving compact remnant, roughtly agrees with observations on the range of ejected mass [32]. White dwarfs in this mass range are known to be primarily oxygen-neon-magnesium mixtures or, at least, to have an oxygen-neon core [17. Although not conclusive, the above considerations support the hypothesis that the progenitors are primarily $\mathrm{O}-\mathrm{Ne}-\mathrm{Mg}$. This possibility has been studied in ref. [33] assuming some unknown external trigger. However, in order to have sufficient energy release from fusion alone, these models boost the fraction of $\mathrm{Ni}^{56}$ in the final state which then produces overly bright explosions. Very close to the Chandrasekhar mass there is a debated suggestion as noted above that the white dwarfs have an iron core which, of course, produces no energy output from fusion but would produce a large amount of energy if there is degeneracy breakdown.

Table1 in connection with the energy sufficiency of carbon-oxygen dwarfs indicates that, if the progenitor nucleus has atomic number 8 or higher, the energy release from fusion alone is inadequate to explain observations and appreciable energy deposition is required from a source beyond the standard model. This could lead to a problem in the binary models with understanding the homogeneity of SN Ia namely the total fusion energy available is sensitive to the composition of the white dwarf; dwarfs with a higher metallicity would produce a significantly smaller amount of $\mathrm{Ni}^{56}$ and/or a significantly lower amount of ejecta kinetic energy contrary to observations. On the other hand, if SN Ia come from a very limited range of white dwarf masses near $M_{C}$, there is also a problem in producing a sufficient rate of supernovae.

As we show below, the phase transition model provides an extra energy input increasing with atomic number. For example, the energies released if the protons in carbon or oxygen convert to scalars dropping into the susy ground state are

$$
\begin{aligned}
& \Delta E(C)=4\left(E_{1 p}-E_{1 s}\right)+6\left(E_{1 s}-\tilde{E}_{1 s}\right) \\
& \Delta E(O)=6\left(E_{1 p}-E_{1 s}\right)+8\left(E_{1 s}-\tilde{E}_{1 s}\right)
\end{aligned}
$$

where $\tilde{E}_{1 s}$ is the energy of the susy $1 s$ state which may or may not be the same as that in normal carbon or oxygen.

The nuclear shell model energies exhibiting the magic numbers have been known since the 1950's, 34] 35] and can be used to determine the excitation energies of various nuclei. A modified harmonic oscillator potential or a modified square well illustrates the approximate equally spaced shells. The harmonic oscillator parameters of the low lying elements, $6 \leq$ $Z \leq 12$, depend on the atomic number but preserve the equal spacing so the excitation energies are scaled relative to those of carbon. We can calibrate the excitation energies using the empirical relation between the $1 s$ ground state to the first excited state in carbon and 
oxygen [36]:

$$
\begin{aligned}
& E_{1 d 5 / 2}(C)-E_{1 s}(C)=4.44 \mathrm{MeV} \\
& E_{2 s}(O)-E_{1 s}(O)=6.05 \mathrm{MeV}
\end{aligned}
$$

The $2 s$ state and the $1 d 5 / 2$ are nearly degenerate being part of the same shell model energy level. The total internal energies of carbon and oxygen relative to the $1 \mathrm{~s}$ ground state, multiplying by a factor of 2 to include neutrons, are then as an example

$$
\begin{aligned}
& E_{C}=2 \cdot 4 \cdot E_{1 p 3 / 2}(C)=12.008 \mathrm{MeV} \\
& E_{O}=2 \cdot\left(4 \cdot E_{1 p 3 / 2}(C)+2 \cdot E_{1 p 1 / 2}(C)\right) \cdot 6.05 / 4.44=26.5 \mathrm{MeV} .
\end{aligned}
$$

In this article, given the present state of observations, we are interested primarily in the sense of the metallicity effects and only roughly in the magnitude of the effects. With this in mind we will assume

$$
E_{n, l}(Z+1)-E_{1 s}(Z+1) \approx\left(E_{n, l}(Z)-E_{1 s}(Z)\right) \cdot 6.05 / 4.44
$$

We can then construct table2 for the energies of the low lying elements relative to the $1 \mathrm{~s}$ ground states.

\begin{tabular}{|c|c|c|}
\hline $\mathrm{Z}$ & element & $\Delta \rho / \rho$ \\
\hline 6 & carbon & $0.0467=0.0078 \cdot Z$ \\
\hline 8 & oxygen & $0.0562=0.0070 \cdot Z$ \\
\hline 10 & neon & $0.0719=0.0072 \cdot Z$ \\
\hline 12 & magnesium & $0.0824=0.0069 \cdot Z$ \\
\hline 26 & iron & $0.204=0.0079 \cdot Z$ \\
\hline
\end{tabular}

Table 2: The fractional change in mass or density of the indicated element on giving up its excitation energy due to degeneracy breakdown as in the susy phase transition. The values for $\Delta \rho / \rho$ include an equal contribution from neutrons. If there is an extra contribution coming from the second terms in eq.5.23, this would also be proportional to the atomic number. We neglect the possible suggestion of quadratic $Z$ dependence.

The main point of table2 (and of this article) is that the susy energy release increases with atomic number while the energy from fusion shown in table1 decreases with Z. This complementarity is the beginning of the susy explanation for the uniformity of the supernovae Ia.

In earlier work on the phase transition model based on the Fermi gas model for even-even nuclei, the Pauli energy was taken to be simply and universally proportional to the density, $\Delta \rho \approx 0.02 \rho$ and a term proportional to a power of $\rho / \rho_{c}$ was added to the action to allow for the possibility of a transition-inhibiting effect analogous to the inhibiting of the liquid to 
gaseous transition at high pressure. In the absence of such a term the action would go to zero as the white dwarf mass approaches $M_{C}$ in which limit the Coleman-DeLuccia model provides no guidance. The white dwarf lifetime defined by eq.4.11 would approach infinity as one approaches the Chandrasekhar mass from below. However, the actual lifetime above a mass of about $1.38 M_{\odot}$ would approach zero due to standard model fusion ignition.

In the nuclear shell model as tabulated in table2, the Pauli excitation energies, neglecting a possible quadratic term, are proportional to the atomic number:

$$
\Delta \rho \approx 0.007 Z \rho
$$

Thus in the Shell Model the critical density in eq.4.16 would be expected to be inversely proportional to the atomic number. We would define a new critical density, $\rho_{c}$, and minimum lifetime, $\tau_{0}$, as free parameters in the fit to the delay time distribution. The action is then parameterized as

$$
B=\left(\frac{\rho_{c}}{\bar{Z} \rho}\right)^{3}+b_{0}\left(\frac{\bar{Z} \rho}{\rho_{c}}\right)^{4 / 3} .
$$

Since white dwarf masses in the range above 0.9 are expected to be progressively $\mathrm{O}-\mathrm{Ne}-\mathrm{Mg}$ mixtures, we write for mean atomic number:

$$
\bar{Z}=7+10(M-0.9)
$$

The calculations, for simplicity, treat a star of fixed atomic number constituents assuming that interpolating between integer $Z$ is a reasonable approximation to a mixture of constituents. The parametrization of eq.5.29 ranges from an average atomic number between that of carbon and oxygen at $M=0.9$ to that of magnesium as $M$ approaches the Chandrasekhar mass. Some $25 \%$ to $30 \%$ of white dwarfs show metal lines in their spectra although how this composition is produced is debated. Perhaps, in an environment enriched by a history of nearby supernova, a main sequence star concentrates higher $Z$ elements near the center and preferentially passes them on to the daughter white dwarf after the lighter elements are blown off. It has also been suggested that spectral evidence for high Z elements in cool white dwarfs could indicate accretion from rocky planets [37, [38].

With the $\mathrm{Z}$ dependent action of eq.5.28 the inverse lifetime for a white dwarf of mass $\mathrm{M}$ is

$$
\tau(M)^{-1}=\frac{1}{\tau_{0}} \frac{V(M)}{V_{\max }}
$$

with now

$$
V(M)=\int d^{3} r e^{-\left(\rho_{c} /(\bar{Z} \rho(r))^{3}-b_{0}\left(\bar{Z} \rho(r) / \rho_{c}\right)^{4 / 3}\right.}
$$

$V(M)$ is an increasing function of $\bar{Z}$ which would make the lifetime a decreasing function of $\bar{Z}$ but this dependence is largely cancelled by the normalization to $V_{\max }$. Relying on the 
observed lack of a compact remnant and observational evidence ref. [39] that accretion is statistically not a major factor in white dwarfs undergoing SN Ia, we can neglect accretion and identify the ejected mass with the progenitor mass at birth. Multiplying by the white dwarf birth mass distribution from section 2 we can write the combined distribution in delay time and progenitor mass.

$$
\frac{d^{2} N}{d t d M}=a_{0} F(M) e^{-t / \tau(M)} / \tau(M)
$$

From eq.5.32 we can derive the SN Ia rates as functions of delay time, $t$, since birth and ejected mass or birth mass. These are

$$
\frac{d N}{d t}=a_{0} \int d M F(M) e^{-t / \tau(M)} / \tau(M)
$$

and

$$
\frac{d N}{d M}=a_{0} F(M) \cdot\left(1-e^{-t_{g} / \tau(M)}\right)
$$

It takes about 0.04 Gyr for a heavy main sequence star to produce a white dwarf so the time since parent star birth and the time since white dwarf birth (defined as the cessation of fusion) differ by about $0.04 G y r$. The $t$ in eq. 5.33 should be replaced by $t-0.04$ if we want $t$ to represent the time since main sequence star formation.

\section{The Delay Time and Ejected Mass Distributions}

The distribution in delay times between white dwarf birth and SN Ia explosion has been measured with several techniques, the most accurate of which is illustrated in the red points of figure 1 of [40]. We do a $\chi^{2}$ minimization of eq.5.33 to these three large bin data points leading to the fit shown in figure 2

The resulting best fit parameters are

$$
\begin{aligned}
& \rho_{c}=58.8 M_{\odot} / R_{E}{ }^{3}=4.5010^{8} \mathrm{~g} / \mathrm{cm}^{3} \\
& \tau_{0}=0.418 \text { Gyr } \\
& b_{0}=0 .
\end{aligned}
$$

Some discussion is warranted here. First of all the best fit is not sharply determined. Good fits with $\chi^{2}$ values less than 1 per degree of freedom are found with $\rho_{c}$ varying by up to $30 \%$ and $b_{0}$ values as large as 1.5. Correlated values of $\tau_{0}$ are found varying by as much as ten percent from the best fit. With respect to the $b_{0}$ values one should note that the Coleman-DeLuccia theory, while not specifying non-leading behavior in the action, is 


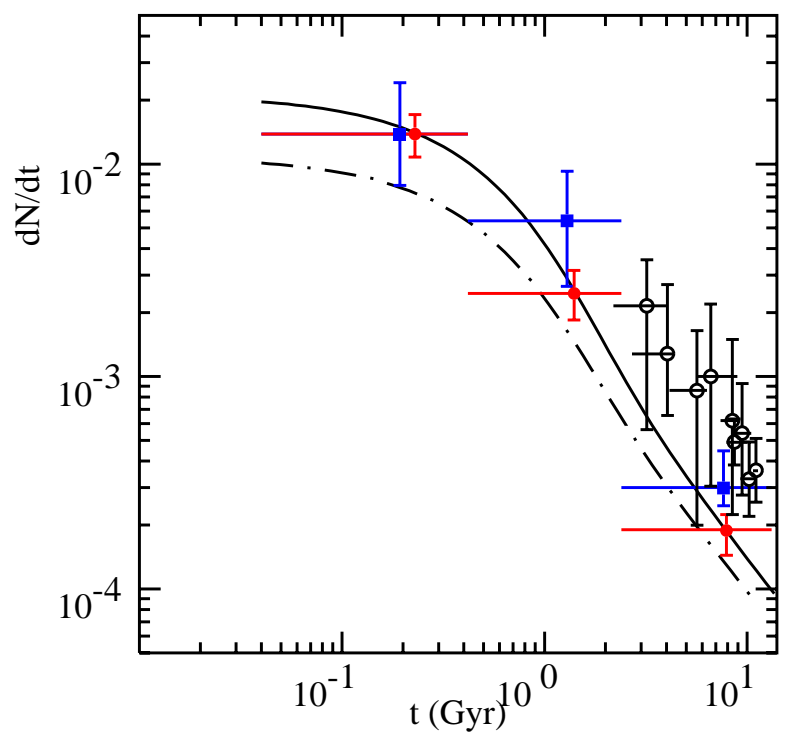

Figure 2: SN Ia delay time distributions. The solid curve shows the DTD for average to high metallicity environments. For low metallicity environments the DTD is reduced as shown in the dot-dashed curve due to the modified parameters of eq.2.7. Data is taken from [40] neglecting earlier data with larger errors.

derived for large action. With $b_{0}=0$, the action approaches zero near the center of high mass white dwarfs. With $b_{0} \approx 0.5$ or larger, the action is everywhere greater than unity. However, since the volume integral for $V(M)$ suppresses the behavior near $r=0$, the theory should be acceptable even for $b_{0}=0$ especially since the predicted supernova progenitor mass distribution shown in figure 3 is suppressed at high mass. In addition, as the white dwarf mass rises above 1.38, nuclei will be within range of the strong interactions so fusion will be ignited preempting the susy phase transition and making the action $B$ irrelevant.

The white dwarf lifetime as a function of its mass is not greatly changed from the plot shown in ref. [6] where there is no explicit Z dependence in the action but, in the present work, the free parameters have been adjusted accordingly.

The delay time distributions, DTD, have a shape that are nearly independent of the host galaxy metallicity although the integrated supernova rate is significantly lower in low metallicity environments. Moreover, supernovae in star-burst galaxies would be expected to have an enhanced rate especially at small delay times. The shape and magnitude of the 
DTD is well reproduced by the phase transition model which also gives a prediction for the behavior at small delay times. The fact that, over a restricted range, the observed DTD plotted on a log-log scale is approximately linear with a slope near the dimensional analysis value of -1 has been widely cited as supporting the double degenerate model [1]. However, the binary models as reviewed there underpredict the DTD and vary among themselves by a factor of 3 to 10 in the low delay time region (see especially fig. 8 of reference [1]).

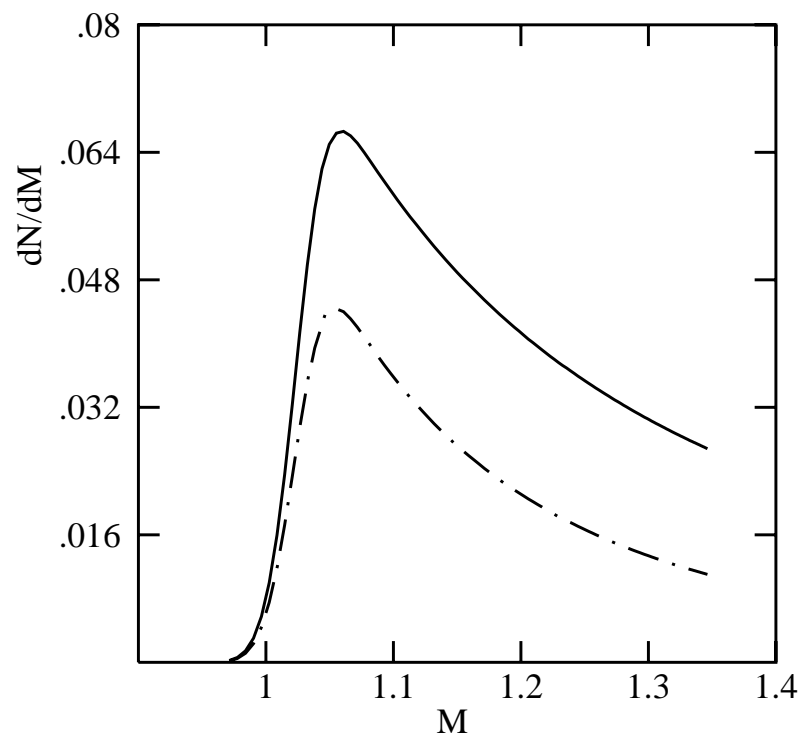

Figure 3: Progenitor mass distribution in high to average metallicity environments (solid curve) and in low metallicity environments (dot-dashed curve). Neglecting the compact surviving remnant these curves represent also the ejected mass distribution predictions.

In the phase transition model the ejected mass should be the same as the progenitor mass since the surviving susy core has a negligible mass. One sees from fig. 3 that the progenitor mass is strongly peaked near one solar mass.

In the double degenerate model for SN Ia it has been suggested that the presence of a third star might be needed to throw two white dwarfs into each other with a short delay time. This possibility reduces the predicted rate and has now been strongly disfavored as a major source of SN Ia [41].

The white dwarf lifetime as a function of its mass is shown in fig. 4. the phase transition model predicts no old white dwarfs in the quasi parabolic region except for exceptional, 
rapidly accreting stars as discussed in ref. [6]. It should be noted that the best fit parameters from the delay time distribution also correctly predict the edge of the white dwarf age vs mass plot in fig. 4 as well as, roughly, the lower edge of the ejected mass distribution as measured in 32 .

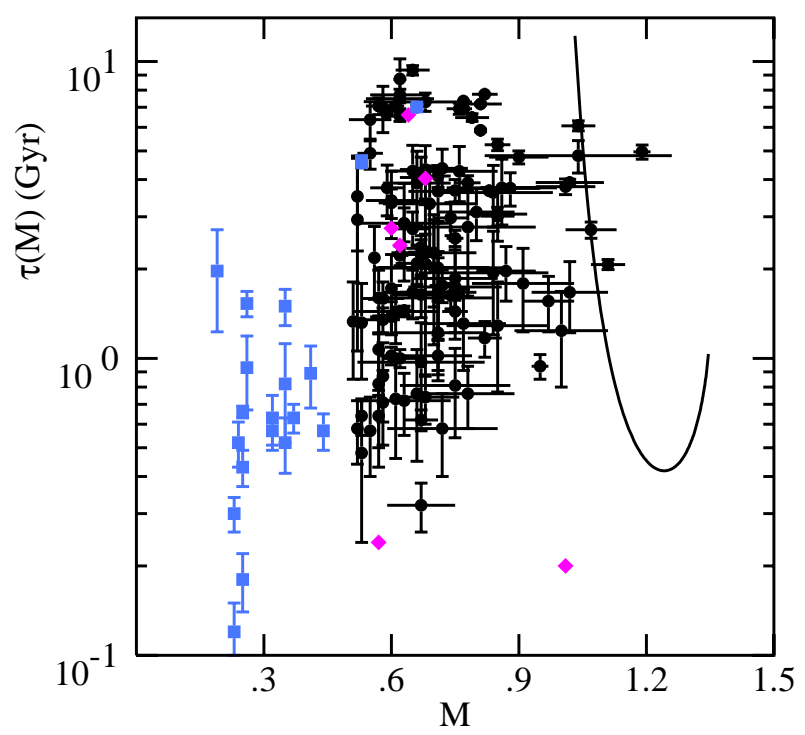

Figure 4: White Dwarf lifetime vs stellar mass in the susy phase transition model. Of the 95 cool DA white dwarfs in the data of [42] those with mass greater than $0.5 M_{\odot}$ are shown in black with measured ages. Of these 95, 19\% "known or suspected" of being in double degenerate binaries are plotted in blue squares as is the one DB white dwarf in this sample. The DB dwarfs have, by definition, a thin helium atmosphere. The uncertainty in the mass of these stars which ranges from $2 \%$ to $50 \%$ is ignored. The present absence of high mass white dwarfs in DD binaries is the major problem facing the DD scenario. It is unknown why the DB stars are so much less likely to be in double degenerate binaries which could be a problem for the double detonation model in which fusion is ignited in an accreting helium layer. The six nearest white dwarfs, shown in magenta, all have lifetimes greater than the current age of the universe.

In terms of the gravitational binding energy, $B$, (not to be confused with the action of eq.4.16), the fusion energy release, $E_{f u s}$, the electron internal energy released when the star explodes, $U$, and the kinetic energy of the ejecta, $E_{k i n}$, we can write the total standard model 
energy deficit

$$
E_{x}=B+E_{k i n}-E_{f u s}-U
$$

These separate energies are calculated in ref. 3] and elsewhere. For instance, as treated there,

$$
E_{\mathrm{kin}}=5.610^{-4}\left(1.22 M_{\odot}+0.2 M\right)
$$

In addition to the decay energy from nickel and other radioactive elements, a small amount of radiated energy, about $1 \% E_{\text {kin }}$, associated with the production of this kinetic energy should be considered as included in $E_{\text {kin }}$. As alluded to above, the standard model energy deficit is consistent with zero in the case of a roughly fifty-fifty mixture of carbon and oxygen. However, since no compact remnants are observed and the ejected mass distribution ranges from $0.9 M_{\odot}$ to $1.4 M_{\odot}$ in which mass range stars are expected to be oxygen-neon-magnesium mixtures, there is a substantial energy deficit in the standard model which we propose is compensated by the susy energy release:

$$
E_{\text {susy }}=E_{x}
$$

The total energy released beyond that required to unbind the star is

$$
E_{t}=E_{\text {susy }}+E_{f u s}+U-B
$$

As stated in the caption of table1, the energy release is that after standardization for the amount of nickel [43] and does not include the decay energy of $\mathrm{Ni}^{56}$. Although the fusion energy release in the full star and the susy energy release in a small core vary rapidly with progenitor mass, the sum of the two grows only slowly, closely tracking the total energy release beyond unbinding. We propose that this is the basis for the uniformity of SN Ia events. The susy core which should shrink to a black hole after cooling has a mass roughly $0.1 \%$ of the progenitor mass:

$$
m_{\text {core }}=\frac{E_{\text {susy }}}{0.007 \cdot \bar{Z}}
$$

In fig.5, it is seen that the susy model predicts, after standardization, about a $5 \%$ variation in total energy release beyond unbinding.

Since, after standardization, the energy release beyond unbinding rises toward higher white dwarf mass and mean atomic number, the model is consistent with the observation [44, [45] that the Hubble residual correlates positively with metallicity. At very low metallicity as would be obtained at high red shift, this effect would need to be taken into effect in determining the apparent Hubble constant.

In the binary models the released energy tracks the more rapidly varying fusion energy making the Phillips relation and the SN Ia uniformity a long-standing challenge. 


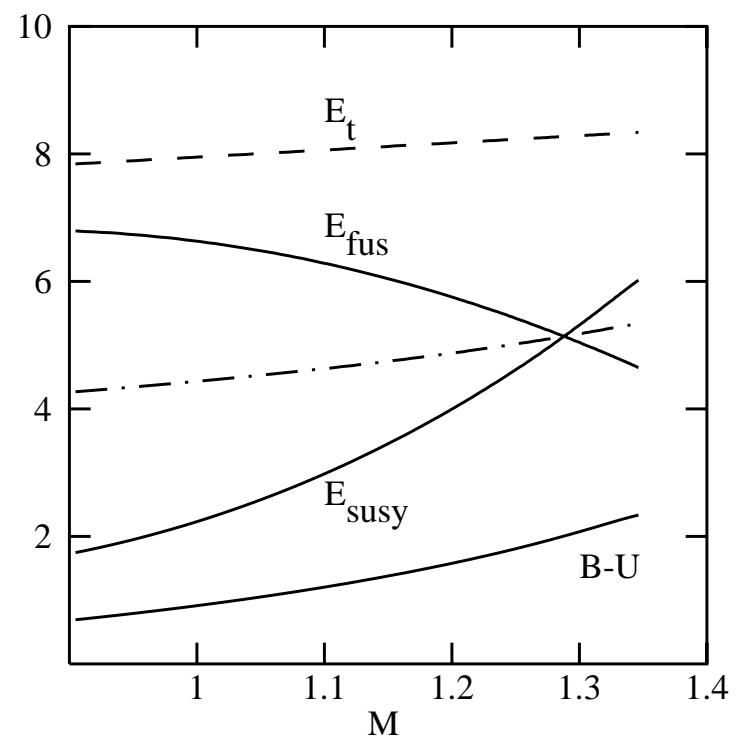

Figure 5: Various energies in SN Ia events in units of $10^{-4} M_{\odot}$ versus ejected mass, $M$, in solar mass units. The curves label the gravitational binding energy minus internal electron energy, $B-U$, the total fusion energy, $E_{f u s}$, released and the nuclear degeneracy energy released in the small core, $E_{\text {susy. }}$. The mean of the fusion energy and released susy energy is shown in the dot-dashed curve. The total released energy, 6.39, beyond that required to unbind the star is shown in the dashed curve.

\section{Summary}

Some forty five years since the binary models for SN Ia were proposed, a review of all the binary models [2] has still not been able to identify a unique progenitor system nor explain how a mixture of widely different progenitor systems can produce the observed homogeneity. We have argued that the possibility of a phase transition mechanism should not be ignored. Going beyond the six earlier indications of radical new physics in SN Ia [3, the host galaxy effects treated here could be taken as supporting the idea that the phase transition responsible for SN Ia is a transition to an exact susy phase. This possible connection is based on the indication that the standard model energy deficit grows with progenitor mass and metallicity as does the degeneracy energy.

With regard to the single degenerate model for $\mathrm{SN}$ Ia, continuing research on this model 
is based on the possibility of somehow avoiding the counter-indications from the $\mathrm{X}$ ray data of [39]. The 5\% Gilfanov-Bogdan limit is based on the inconsistency, given $\mathrm{X}$ ray constraints, of $1.2 M_{\odot}$ white dwarfs accreting to the Chandrasekhar mass. The conflict is exacerbated if the progenitors are initially below one solar mass, as required if they are C-O dwarfs, since then an even higher accretion rate would be required.

In the double degenerate model, continuing research requires the expectation that a substantial population of high mass white dwarf binaries will be discovered above that suggested by the Salpeter initial mass function and significantly above the $20 \%$ "known or suspected" in the sample of ref. [42].

In the models of Sim et al. [4 the trigger is assumed to provide negligible energy release to supplement the available fusion energy. We have argued that the actual composition should be $\mathrm{O}-\mathrm{Ne}-\mathrm{Mg}$ with the reduction in available fusion energy being compensated by the degeneracy energy released in a small core. The peak in the progenitor mass distribution, shown in figure 3, is in reasonable agreement with one of the Sim et al. models and the range of progenitor masses roughly agrees with observations [32]. The delay time distribution is also well reproduced as in fig.2. Although we have found in ref. [6] that accretion, in the susy model, is generally not important in supernova production, a small accretion rate $\left(\approx 10^{-11} M_{\odot} / y r\right)$ onto high mass white dwarfs could be effective in producing the $1 \%$ of super-Chandrasekhar events and the second peak in the ejected mass distribution possibly suggested in ref. [32]. The super-Chandrasekhar events, of course, would require some temporary stabilization effect such as the spin-up/spin-down model [46]. The tail of the Salpeter prediction of the white dwarf mass distribution above a mass of 1.38 will also immediately produce a supernova since, above this mass, the nuclei will be within the range of the strong interactions. This could also contribute up to $1 \%$ of supernovae.

In the ejected mass distribution and the delay time distribution, the metallicity dependence should be visible in the future high statistics supernova data expected from the Gaia collaboration which should also settle the question as to the possible existence of high mass double degenerate systems [47]. Gaia has already succeeded in identifying binary companions among the progenitors of core collapse supernovae [48] but has not yet reported observations of binary progenitors of SN Ia. When the double mass distribution and other orbital distributions of white dwarf binaries are in hand, the DD scenario, if correct, should be able to produce delay time distributions and ejected mass distributions to compare with those of the phase transition model.

The minimum energy released in the nucleation of a critically sized bubble is

$$
E_{\text {susy }}(\min )=\overline{\Delta \rho} \cdot \frac{4 \pi}{3} R_{c}{ }^{3}=36 \pi^{3} S^{3} / \overline{\Delta \rho}^{2}
$$

where $R_{c}$ is the critical radius of eq.4.14. The fit to the critical density implies that the surface tension is

$$
S \approx 2 \cdot 10^{-21} M_{\odot} / R_{E}{ }^{2}
$$


The minimum bubble size from eq.4.14 is then small on the stellar scale but large compared to the inter-atomic scale. The much larger full susy energy released depends on the final radius of the susy bubble.

The growth of the susy bubble is halted when the critical radius becomes greater than its current radius as could happen due to a sharp falloff of density or to its entrance into a region of lower atomic number. The supernova explosion is expected to create a cavity of density significantly lower than that of interstellar space. The extent of a white dwarf iron core as discussed above, could also be critical since iron is inert to fusion but would provide a large energy release if there is degeneracy breakdown as seen in table 2 . In eq.5.29 we have tentatively assumed that the mean atomic number never increases above that of magnesium even if a small iron core builds up in the center.

If, on the other hand, the iron core becomes a non-negligible fraction of the total, fusion will be greatly suppressed and the observed sub-luminous events (SN Iax) may be produced. These would be predicted to have ejected mass near the Chandrasekhar limit and might be contained in the high ejected mass events of ref. [32].

Prior to consideration of the host galaxy correlations, indications of the need for radical new physics could be satisfied by other types of phase transitions such as transitions to a quark-gluon plasma although no such model has as yet been worked out for SN Ia. The observation that the explosion energy increases with metallicity as does the degeneracy energy is in line with exact susy being the phase transition final state. Finally, it must be noted that the hypothesis of a phase transition to an exact susy phase implies that the broken susy phase must also exist and susy particles must be found at sufficiently high accelerator energies if the model suggested here is to be viable.

\section{References}

[1] D. Maoz, F. Mannucci, and G. Nelemans, Observational Clues to the Progenitors of Type Ia Supernovae, DOI:10.1146/annurev-astro-082812-141031, (arXiv:1312.0628v3).

[2] M. Livio and P. Mazzali, On the Progenitors of Type Ia Supernovae Physics Reports, 736, 1-23 (2018), (arXiv:1802.03125).

[3] L. Clavelli ,Six Indications of Radical New Physics in SN Ia, IJMPA 32, No. 31 (2017), (arXiv:1706.03393).

[4] S. Sim et al., Detonations in sub-Chandrasekhar $C+O$ white dwarfs, Astrophys. J. Letters 714 L52-L57 (2010), (arXiv:1003.2917).

[5] P. Biermann and L. Clavelli, A supersymmetric model for triggering Supernova 1a in isolated White Dwarfs, Phys. Rev. D 84,023001 (2011), (arXiv:1011.1687v2). 
[6] L. Clavelli,Degeneracy Breakdown as a Source of Supernovae Ia, Journ. of Cosmol. \& Astropart. Phys.1801 (2018) no.01, 052 (2018-01-30), (arXiv:1609.02742).

[7] S. Blondin et al., Evidence for sub-Chandrasekhar-mass progenitors of Type Ia supernovae at the faint end of the width-luminosity relation, Mon.Not.Roy.Astron.Soc. 470 (2017) no.1, 157-165, (arXiv:1706.01901).

[8] A.C. Calder, B.K. Krueger, A.P. Jackson, and D.M. Townsley, The Influence of Chemical Composition on Models of Type Ia Supernovae, Front. Phys. (Beijing) 8, 168 (2013) (arXiv:1303.2207).

[9] K.A. Williams, M. Bolte, K. Koester, An Empirical Initial-Final Mass Relation from hot, massive White Dwarfs in NGC2168 (M35), Astrophys. J. 615, L49 (2004).

[10] K. Nomoto, Evolution of 8-10 $M_{\odot}$ stars toward electron capture supernovae: Formation of electron degenerate $\mathrm{O}+\mathrm{Ne}+\mathrm{Mg}$ cores, Astrophys. J. 277, 791 (1984).

[11] S.E. Woosley, A Heger, T.A. Weaver, The Evolution and Explosion of Massive Stars, Rev. Mod. Phys. 74 (4), 1015 (2002).

[12] cronodon.com/Spacetech/WhiteDwarf.html.

[13] J.A. Panei, L.G. Althaus, O.G. Benvenuto, Evolution of iron core white dwarfs, Astron. \& Astroph. 353, 970 (2000) (astro-ph/9911371).

[14] J. Madej et al., Theoretical UBVRI colors of Iron Core White Dwarfs, Astron. \& Astroph. 412, 847 (2003) (astro-ph/0308184).

[15] J.L. Provencal et al., Testing the White Dwarf Mass-Radius Relation with Hipparcos, Astrophys. J. 494, 759 (1998).

[16] B.W. Carroll and D.A. Ostlie, An Introduction to Modern Astrophysics, AddisonWesley (1996).

[17] J. Madej, M. Nalezyty, and L.G. Althaus, Mass distribution of DA white dwarfs in the First Data Release of the Sloan Digital Sky Survey, Astron. \& Astroph. 419, L5 (2004) (astro-ph/0404344).

[18] M. Geha et al., Stellar IMF of ultra faint dwarf galaxies: Evidence for IMF variation, Astrophys. J. 771, 29 (2013), (arXiv:1304.7769).

[19] P. Kroupa et al., On the variation of the IMF, Month. Not. Roy. Astr. Soc. 322, 231 (2009) (astro-ph/0009005).

[20] X. Meng et al.,Initial-Final Mass Relationship for Stars of Different Metallicities , Astron. \& Astroph. 487, 625 (2008) (arXiv:0710.2397) . 
[21] S. Coleman, Phys. Rev. D15, 2929 (1977); C.G. Callan, \& S. Coleman, Phys. Rev. D16, 1762 (1977); S. Coleman, and F. DeLuccia, Gravitational Effects on and of Vacuum Decay, Phys. Rev. D 21, 3305 (1980).

[22] A.S. Gorsky, \& V.G. Kiselev, False vacuum decay induced by dense matter in twodimensions, Phys. Lett. B304, 214 (1993) .

[23] M.B. Voloshin, Catalyzed decay of false vacuum in four-dimensions, Phys.Rev. D49, 2014-2018 (1994).

[24] K. Konishi et al., Dependences of Type Ia Supernovae Lightcurve Parameters on the Host Galaxy Star Formation Rate and Metallicity, arXiv:1101.4269.

[25] E. Churazov et al., Cobalt-56 gamma-ray emission lines from the type Ia supernova 2014J, Nature 512, 406 (2014), arXiv:1405.3332.

[26] M.J. Childress et al., Measuring nickel masses in Type Ia Supernovae using cobalt emission in nebular phase spectra, /MNRAS 454, 3816 (2015),(arXiv:1507.02501).

[27] P. Hoeflich, J.C. Wheeler, F.K. Thielemann, Type Ia Supernovae: Influence of the initial composition on Nucleosynthesis, Light Curves, Spectra and Consequences for the Determination of $\Omega_{M}$ and $\Lambda$, Astrophys. J. 495, 617 (1998), (astro-ph/9709233).

[28] F. K. Röpke and W. Hillebrandt, The case against the progenitor's carbon to oxygen ratio as a source of peak luminosity variations in Type Ia supernovae, Astron. \& Astroph. 420, L1 (2004), (astro-ph/0403509).

[29] W. Hillebrandt, J.C. Niemeyer, and M. Reinecke, Type Ia Supernova Explosion Models: Homogeneity versus Diversity, AIP Conf. Proc. 522, 53 (2000), (astro-ph/0005584).

[30] N. Giammichele et al.,A large oxygen dominated core from the seismic cartography of a pulsating white dwarf, Nature online 2018, doi:10.1038/nature 25136.

[31] J. Isern et al., On the internal composition of white dwarfs, Nucl. Phys. A688, 122c-125c (2001).

[32] R.A. Scalzo, A.J. Ruiter, and S.A. Sim,Ejected mass dist of SN Ia: a significant rate of non-Chandra-mass progenitors, Month. Not. Roy. Astr. Soc. , 445, 2535 (2014), (arXiv:1408.6601).

[33] K.S. Marquardt et al., Type Ia Supernovae from exploding O-Ne White Dwarfs, Astron. \& Astroph. 580, A118 (2015), (arXiv:1506.05809).

[34] M.A. Preston, Physics of the Nucleus, Addison-Wesley Publishing Company Inc., Reading Massachusetts, (1962).

[35] Bakken et al., https://commons.wikimedia.org/w/index.php?curid=15039395. 
[36] www.tunl.duke.edu/nucldata/html.

[37] A. Kawka et al., Abundance analysis of DAZ White Dwarfs, AIP Conf. Proc. 1331, 238 (2011), (arXiv:1012.2639).

[38] B. Zuckerman et al., Ancient Planetary Systems are orbiting a large fraction of White Dwarf Stars, Astrophys. J. 722, 725 (2010), (arXiv:1007.2252).

[39] M. Gilfanov and Á. Bogdán, An upper limit on the contribution of accreting white dwarfs to the type Ia supernova rate, Nature 463, 924 (2010).

[40] D. Maoz, F. Mannucci, and T. Brandt, Delay Time Distribution of SN Ia from SDSS II, Month. Not. Roy. Astr. Soc. 426, 3282 (2012), (arXiv:1206.0465).

[41] S. Toonen, H.B. Perets, and A.S. Hamers, The rate of WD-WD head on collisions in isolated triplets is too low to explain standard type II SN, (arXiv:1709.00422).

[42] P. Bergeron, S.K. Leggett and M.T. Ruiz, Photometric and spectroscopic analysis of cool white dwarfs with trigonometric parallax measurements, Astrophys. J. Suppl. 133 (2001) 413, (astro-ph/0011286).

[43] M. M. Phillips, The absolute magnitudes of Type IA supernovae, Astrophys. J. Letters 413, L105 (1993).

[44] J.S. Gallagher et al., Supernovae in early-type galaxies: Directly connecting Age and Metallicity with Type Ia Luminosity Astrophys. J. 685, 752 (2008), (arXiv:0805.4360).

[45] Y.-C. Pan et al., The host Galaxies of PTF SN Ia, Month. Not. Roy. Astr. Soc. 438, 1391 (2014), (arXiv:1311.6344).

[46] R. Di Stefano et al., Spin-Up/Spin-Down Models for Type Ia Supernovae, Astrophys. J. 738, L1 (2011).

[47] Brevik et al., Characterizing accreting double white dwarf binaries with Lisa and Gaia, (arXiv:1710.08370).

[48] D. Boubert et al., Binary companions of nearby supernova remnants found with Gaia, Astron. \& Astroph. 606 A14 (2017), (arXiv:1704.05900). 